\title{
Hallazgos de SS-OCT y OCT-A en pacientes con anemia de células falciformes comparados con individuos sanos
}

\section{Findings by SS-OCT and OCT-A in patients with sickle cell disease compared to healthy individuals}

\author{
Diego Bueso-Ponce ${ }^{1}$, Juan Unigarro ${ }^{1}$, Sofía Vidal ${ }^{1}$, Carlos Salgado ${ }^{1}$, Enrique Ramos ${ }^{2}$ y \\ Carlos Abdala-Caballero ${ }^{1 *}$ \\ ${ }^{1}$ Departamento de Retina y Vitreo, Grupo oftalmológico Abdala-Figuerola/Clínica Unidad Laser del Atlántico, Barranquilla; ${ }^{2}$ Departamento de \\ Epidemiología y Bioestadística, Universidad del Sinú, Cartagena. Colombia
}

\section{Resumen}

Objetivo: Describir los cambios en el grosor macular central, el grosor macular promedio, el grosor coroideo central y el área de la zona avascular foveal en los plexos superficial y profundo de pacientes con anemia de células falciformes, $y$ comparar los resultados con un grupo de sujetos sanos. Método: Estudio descriptivo de hallazgos de tomografía de coherencia óptica swept source (SS-OCT) y de angiografía por tomografía de coherencia óptica (OCT-A) en 28 ojos de 14 pacientes con anemia de células falciformes confirmado por electroforesis y 60 ojos de 30 personas sanas sin antecedentes patológicos sistémicos ni oculares. Resultados: En el grupo de anemia de células falciformes la mediana del grosor macular central fue de $194 \mu \mathrm{m}$, frente a $229 \mu \mathrm{m}$ en el grupo de personas sanas $(p<0.0001)$. La mediana de zona avascular foveal en el plexo superficial del grupo de anemia de células falciformes fue de $0.347 \mathrm{~mm}^{2}$, frente a $0.243 \mathrm{~mm}^{2}$ en el grupo sano, y en el plexo vascular profundo la mediana fue de 0.461 frente a $0.321 \mathrm{~mm}^{2}$, respectivamente $(p<0.0001)$. Las medidas comparativas del grosor macular promedio y del grosor coroideo no demostraron diferencia estadísticamente significativa entre ambos grupos. Conclusiones: En comparación con el grupo de sanos, los pacientes con anemia de células falciformes presentaron diferencias estadisticamente significativas: menor grosor macular central y aumento del área de la zona avascular foveal en los plexos superficial y profundo. La SS-OCT y la OCT-A son modalidades de estudio no invasivo que proporcionaron imágenes de alta resolución que podrían ser útiles para detectar cambios anatómicos estructurales y microvasculares en ambos plexos en pacientes con anemia de células falciformes.

Palabras clave: Anemia de células falciformes. Angiografía por tomografía de coherencia óptica. Plexo capilar profundo. Plexo capilar superficial. Tomografía de coherencia óptica swept source. Zona avascular foveal.

\section{Abstract}

Purpose: To describe the findings in central macular thickness, mean macular thickness, central choroidal thickness, and foveal avascular zone at the superficial and deep retinal plexus, and to compare the results with a group of healthy subjects. Methods: Descriptive study about SS-OCT and OCT-A findings in 28 eyes (14 patients) with electrophoretic confirmation of sickle cell disease and 60 eyes (30 patients) without systemic or ocular disorders. Results: In the sickle cell disease group,

Correspondencia:

${ }^{*}$ Carlos Abdala

Cra 30, Corredor Universitario 1-850

Médica, Consultorio 812

Fecha de recepción: 21-05-2020

Fecha de aceptación: 12-08-2020

E-mail: cabdala@ retinayvitreo.com
Disponible en internet: 13-01-2021

Rev Mex Oftalmol. 2021:95(2):56-62

www.rmo.com.mx 0187-4519/O 2020 Sociedad Mexicana de Oftalmología. Publicado por Permanyer. Este es un artículo open access bajo la licencia CC BY-NC-ND (http://creativecommons.org/licenses/by-nc-nd/4.0/). 
median central macular thickness was 194 vs. $229 \mu \mathrm{m}$ in the healthy subjects group $(p<0.0001)$. The area of the foveal avascular zone at the superficial plexus showed a median value of $0.347 \mathrm{vs.} 0.243 \mathrm{~mm}^{2}(p<0.0001)$ and at the deep capillary plexus of 0.461 vs. $0.321 \mathrm{~mm}^{2}$ in the sickle cell disease and healthy subjects groups, respectively $(p<0.0001)$. The comparative measures of mean macular thickness and central choroidal thickness did not reveal significant statistical differences between groups. Conclusions: Compared to a healthy control group, patients with sickle cell disease had a statistically significant lower central macular thickness and an enlarged foveal avascular zone at both the superficial and deep plexuses. SS-OCT and OCT-A are non-invasive imaging modalities that provide high resolution images that can be useful to detect anatomic and micro vascular alterations in patients with sickle cell disease.

Key words: Sickle cell disease. Optical coherence tomography angiography. Deep capillary plexus. Superficial capillary plexus. Swept source optical coherence tomography. Foveal avascular zone.

\section{Introducción}

La anemia de células falciformes (ACF) es una hemoglobinopatía de herencia autosómica recesiva que se caracteriza por la presencia de hemoglobina $S$ como resultado de la mutación puntual de un aminoácido, en la cual se sustituye el ácido glutámico por valina en la posición 6 de la cadena $\beta$-globina ${ }^{1,2}$.

Fue descrita en 1910 por Herrick en un paciente del oeste de India ${ }^{3}$. En Colombia se considera como una enfermedad huérfana y su incidencia es de 0.03/100,000 habitantes ${ }^{4}$. Se estima que en los Estados Unidos de América existen 100,000 individuos afectados por la enfermedad, y la prevalencia es mayor en el Medio Este, el Mediterráneo, el suroeste de Asia y África subsahariana ${ }^{5}$.

Las manifestaciones oftalmológicas son comunes, pero en muchos casos son subdiagnosticadas. La viscosidad sanguínea, la estructura anormal y la fragilidad de los eritrocitos desencadenan hemólisis, oclusiones vasculares a repetición y daño endotelial que lleva a isquemia de la red capilar retiniana, lo cual estimula la neovascularización 0 atrofia retiniana ${ }^{2}$.

Por más de 40 años la angiografía fluoresceínica ha sido el método de referencia para el estudio de la anatomía vascular retiniana en pacientes con $\mathrm{ACF}^{6,7}$. En general predominan los hallazgos periféricos; se han descrito zonas de isquemia en la mácula, irregularidad de la zona avascular de la fóvea (ZAF), microaneurismas y aplanamiento de la excavación foveal ${ }^{8}$.

El advenimiento de la angiografía por tomografía de coherencia óptica swept source (SS-OCT) y de la angiografía por tomografía de coherencia óptica (OCTA) ha permitido estudiar la anatomía vascular retiniana al producir imágenes segmentadas de alta resolución de los plexos superficiales y profundos de la retina ${ }^{9,10}$.

El propósito del presente estudio es describir los cambios en el grosor macular central (GMC), el grosor coroideo central (GCC), el grosor macular promedio
(GMP) y el área de la ZAF superficial y profunda, en pacientes con ACF, y comparar los resultados con los de un grupo de personas sanas.

\section{Método}

Se realizó un estudio descriptivo con un grupo de pacientes diagnosticados de ACF y otro grupo de personas sanas, que fueron valorados en la Clínica Unidad Laser del Atlántico en Barranquilla, Colombia. Se obtuvo la aprobación del comité de ética institucional, todos los participantes firmaron el consentimiento informado y el estudio se adhirió a los lineamientos estipulados en la declaración de Helsinki.

En el grupo de ACF se incluyeron pacientes con hemoglobina tipo SC y SS, confirmado por electroforesis, sin otros antecedentes sistémicos y sin retinopatía proliferativa. Se excluyeron los pacientes con antecedentes de hipertensión arterial, diabetes mellitus, glaucoma, vasculopatías retinianas, tracción vitreomacular, desprendimiento de retina, antecedentes de cirugías vitreorretinianas o imágenes de SS-OCT y OCT-A de mala calidad que no permitieran realizar medidas confiables. En el otro grupo se incluyeron personas sanas sin antecedentes patológicos sistémicos ni oculares, con edades similares a las de los pacientes con ACF.

A todos los pacientes se les realizó una valoración oftalmológica completa. Las imágenes se tomaron con el equipo de estudio Triton Plus DRI OCT (Topcon Corporation, Tokio, Japón) con tecnología SS-OCT y protocolo de mácula 3-D, y OCT-A de $4.5 \times 4.5 \mathrm{~mm}$ con el mismo equipo. Se midió el GMC, el GCC y el GMP utilizando el círculo central de la cuadrícula ETDRS (Early Treatment Diabetic Retinopathy Study) incorporada en el software. La medida de la ZAF se realizó manualmente y por un solo operador, a nivel del plexo vascular superficial y profundo, con la plantilla de colores utilizada para medir la densidad vascular. La medida del área de la ZAF se delineó siguiendo el 
Tabla 1. Características generales y comparación de los resultados entre el grupo con anemia de células falciformes y el grupo control de sujetos sanos

\begin{tabular}{|c|c|c|c|}
\hline & ACF (n = 28) & Sanos $(n=30)$ & $\mathbf{p}$ \\
\hline Edad en años, mediana (RIC) & $21(15-28)$ & $27(23-30)$ & 0.0020 \\
\hline$<20$ años & $14(50.0)$ & $4(6.7)$ & $<0.0001$ \\
\hline 21-30 años & $12(42.9)$ & $42(70.0)$ & 0.0148 \\
\hline $31-40$ & $0(0.0)$ & $0(0.0)$ & -- \\
\hline $41-50$ & $0(0.0)$ & $0(0.0)$ & -- \\
\hline $51-60$ & $2(7.1)$ & $14(23.3)$ & 0.0804 \\
\hline$>60$ & $0(0.0)$ & $0(0.0)$ & -- \\
\hline $\begin{array}{l}\text { Sexo } \\
\text { Femenino } \\
\text { Masculino }\end{array}$ & $\begin{array}{l}18(64.3) \\
10(35.7)\end{array}$ & $\begin{array}{l}40(66.7) \\
20(33.3)\end{array}$ & 0.8262 \\
\hline $\begin{array}{l}\text { Resultados SS-OCT, media (RIC) } \\
\text { Grosor macular central } \\
\text { Grosor coroideo central } \\
\text { Grosor macular promedio } \\
\text { ZAF superficial } \\
\text { ZAF profunda }\end{array}$ & $\begin{array}{c}194(180-223) \\
281(220-319) \\
289(271-297) \\
0.347(0.266-0.452) \\
0.461(0.376-0.583)\end{array}$ & $\begin{array}{c}229(222-250) \\
292(220-333) \\
280(270-288) \\
0.243(0.198-0.305) \\
0.321(0.244-0.379)\end{array}$ & $\begin{array}{c}<0.0001 \\
0.4490 \\
0.0234 \\
<0.0001 \\
<0.0001\end{array}$ \\
\hline
\end{tabular}

RIC: rango intercuartílico; SS-OCT: tomografía de coherencia óptica swept source; ZAF: zona avascular de la fóvea.

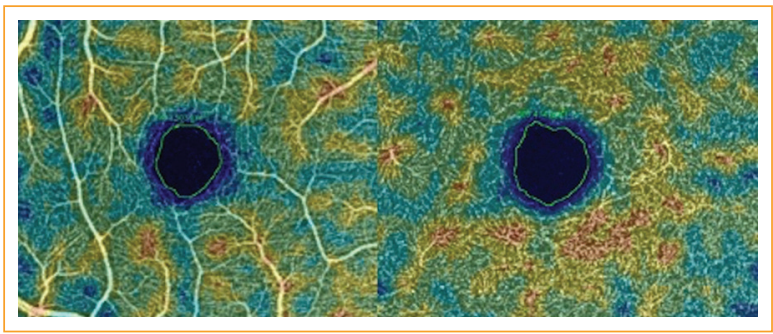

Figura 1. Medida manual del área de la zona avascular de la fóvea a nivel de los plexos vasculares superficial y profundo en una persona sana. Utilizando el mapa de densidad vascular, se trazó el contorno de la zona azul oscuro-negra que corresponde a una densidad vascular del $0 \%$.

contorno del color azul oscuro que codifica para una densidad vascular del $0 \%$ (Fig. 1).

En toda la población seleccionada se tomaron las variables generales, como edad y sexo. El análisis estadístico descriptivo consistió en las variables cualitativas en cálculo de frecuencias absolutas y relativas, mientras que en las cuantitativas se obtuvieron medidas de tendencia central tipo mediana con su respectiva medida de dispersión de rango intercuartílico (RIC), utilizada por la distribución no paramétrica de las variables cuantitativas estimada por la prueba de
Shapiro-Wilk. Las comparaciones de los grosores y las medidas de SS-OCT y OCT-A entre los pacientes con ACF y las personas sanas se realizó con las prueba $U$ de Mann Whitney, y se consideró un valor de $p<0.05$ como diferencia estadísticamente significativa.

\section{Resultados}

Se incluyeron 88 ojos: 28 de 14 pacientes con ACF (cinco con hemoglobina SC y nueve con hemoglobina SS) y 60 de 30 personas sanas. La mediana de edad en el grupo con ACF fue de 21 años (RIC: 15-28) y en los sanos fue de 27 años (RIC: 23-30) ( $p=0.0020)$. En cuanto a la distribución por sexos, el $64.3 \%$ de los pacientes con ACF eran hombres y en las personas sanas la proporción fue del $66.7 \%(p=0.8262)$ (Tabla 1).

Al comparar los resultados de la SS-OCT se encontró para el GMC una mediana en el grupo con ACF de $194 \mu \mathrm{m}$ (RIC: 180-223), y en el grupo sano fue de $229 \mu \mathrm{m}$ (RIC: 222-250) ( $\mathrm{p}<0.0001)$. El GCC mostró medianas en los grupos ACF y sano de 281 y $292 \mu \mathrm{m}$, respectivamente $(p=0.4490)$. El GMP tuvo una mediana de $289 \mu \mathrm{m}$ (RIC: 271-297) en el grupo con ACF y de $280 \mu \mathrm{m}$ (RIC: $270-288)$ en el grupo sano $(p=0.0234)$ (Fig. 2). El área de la ZAF superficial en el grupo con ACF tuvo una mediana de $0.347 \mathrm{~mm}^{2}$ (RIC: 0.266 0.452 ), mientras que en los sanos fue de $0.243 \mathrm{~mm}^{2}$ 


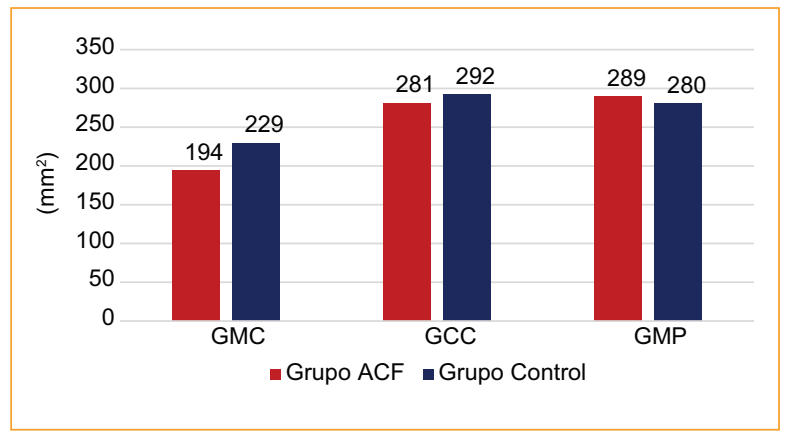

Figura 2. Comparación del grosor macular central (GMC), el grosor coroideo central (GCC) y el grosor macular promedio (GMP) en el grupo de pacientes con anemia de células falciformes (ACF) y en el grupo control de personas sanas (micras).

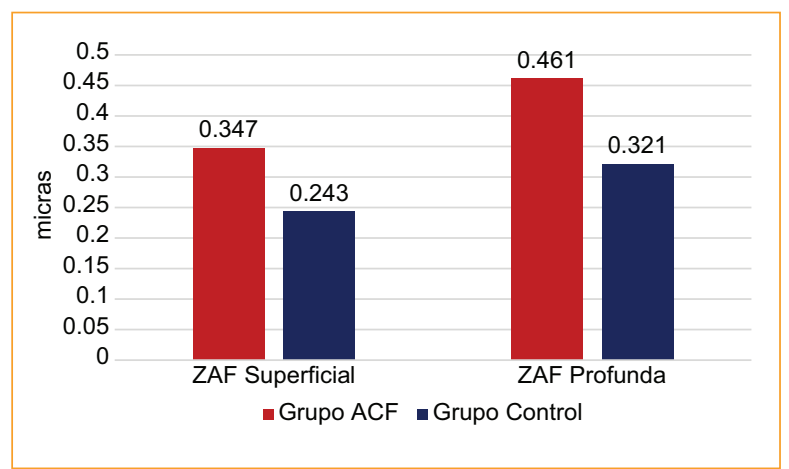

Figura 3. Comparación del área de la zona avascular de la fóvea (ZAF) a nivel de los plexos superficial y profundo en el grupo de pacientes con anemia de células falciformes (ACF) y en el grupo control de personas sanas $\left(\mathrm{mm}^{2}\right)$.

(RIC: $0.198-0.305)(p<0.0001)$. Igual comportamiento se observó en la ZAF profunda, que tuvo una mediana de $0.461 \mathrm{~mm}^{2}$ en el grupo con ACF y de $0.321 \mathrm{~mm}^{2}$ en el grupo sano ( $p<0.0001)$ (Fig. 3). En los pacientes con ACF, la ZAF tuvo un mayor ensanchamiento a nivel del plexo vascular profundo, que se demostró en un paciente en particular con una medida de $0.869 \mathrm{~mm}^{2}$ (Fig. 4).

Al analizar las imágenes de B-scan de la SS-OCT en conjunto con las imágenes de segmentación de los plexos vasculares en la OCT-A, en 10 de 28 ojos (35\%) del grupo de ACF se identificaron zonas de aplanamiento de la excavación foveal, atrofia retiniana temporal a la fóvea y alteración del paralelismo de las capas retinianas que se correlacionaba con zonas

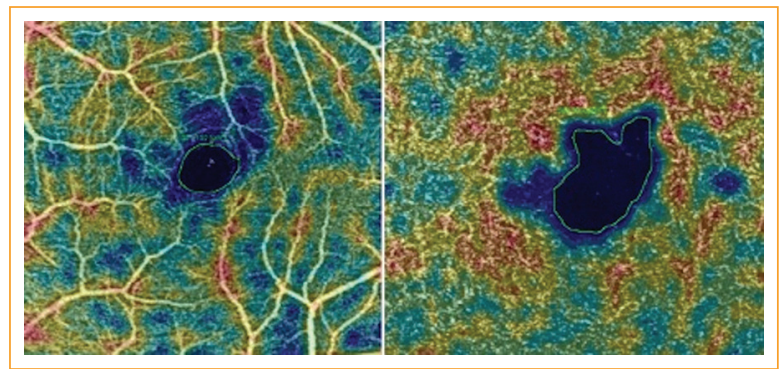

Figura 4. Comparación del aumento del área de la zona avascular de la fóvea profunda con respecto a la superficial.

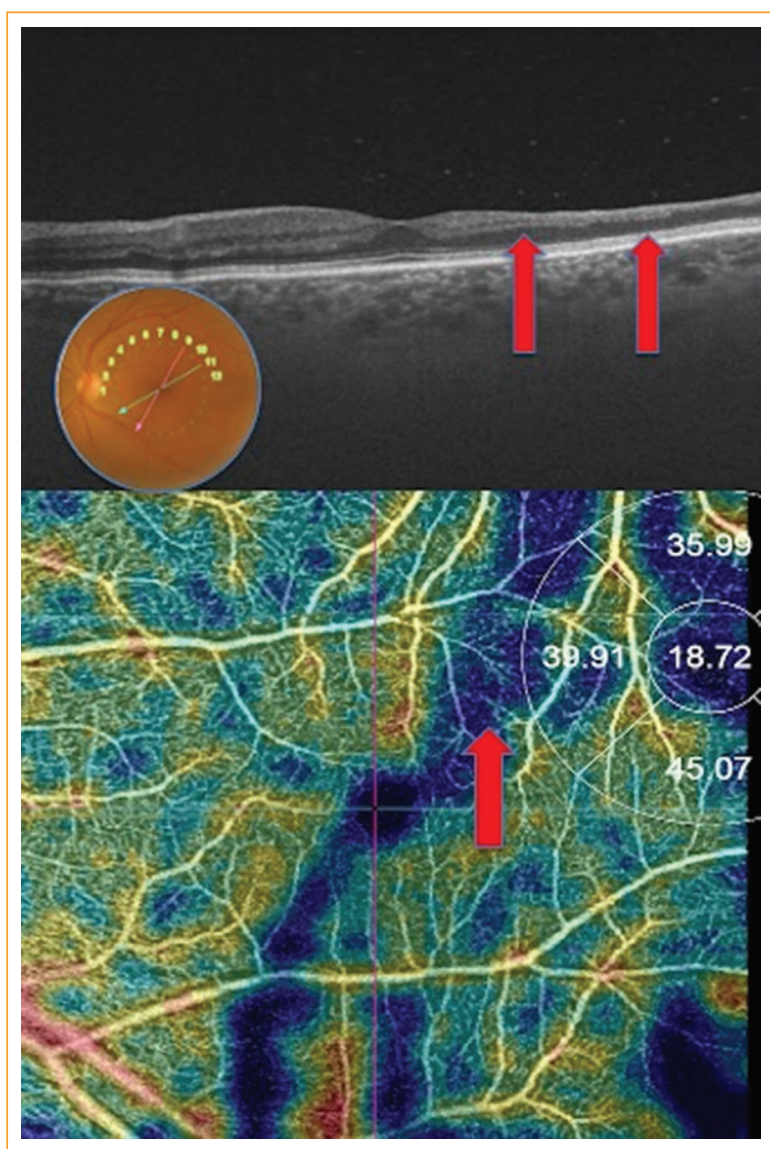

Figura 5. B-scan con imagen correspondiente de angiografía por tomografía de coherencia óptica que muestra las zonas de pérdida de densidad vascular a nivel del plexo profundo asociadas a zonas de atrofia y adelgazamiento retiniano (flechas rojas).

focales de disminución de la densidad vascular y áreas detectadas con el equipo como de no perfusión (Fig. 5).

Con respecto al estado funcional de los pacientes del grupo de ACF, la agudeza visual (AV) sin corrección fue en promedio de 20/25; al realizar el examen de AV 
Tabla 2. Grupo con anemia de células falciformes: comparación entre hallazgos estructurales y agudeza visual

\begin{tabular}{|c|c|c|c|c|c|c|c|}
\hline Paciente & Edad & Ojo & GMC ( $(\mu \mathrm{m})$ & ZAF superficial $\left(\mathrm{mm}^{2}\right)$ & ZAF profunda $\left(\mathrm{mm}^{2}\right)$ & AVSC & AV con estenopeico \\
\hline \multirow[t]{2}{*}{1} & 54 & OD & 223 & 0.228 & 0.941 & $20 / 30$ & $20 / 20$ \\
\hline & & 이 & 245 & 0.221 & 0.281 & $20 / 30$ & $20 / 20$ \\
\hline \multirow[t]{2}{*}{2} & 28 & OD & 178 & 0.362 & 0.525 & $20 / 20$ & \\
\hline & & 이 & 174 & 0.391 & 0.867 & $20 / 20$ & \\
\hline \multirow[t]{2}{*}{3} & 8 & OD & 221 & 0.243 & 0.433 & $20 / 30$ & $20 / 20$ \\
\hline & & 이 & 205 & 0.271 & 0.278 & $20 / 30$ & $20 / 20$ \\
\hline \multirow[t]{2}{*}{4} & 30 & OD & 210 & 0.531 & 0.64 & $20 / 25$ & $20 / 20$ \\
\hline & & 이 & 192 & 0.551 & 0.618 & $20 / 25$ & $20 / 20$ \\
\hline \multirow[t]{2}{*}{5} & 8 & OD & 170 & 0.329 & 0.379 & $20 / 25$ & $20 / 20$ \\
\hline & & 이 & 220 & 0.354 & 0.411 & $20 / 25$ & $20 / 20$ \\
\hline \multirow[t]{2}{*}{6} & 27 & OD & 193 & 0.288 & 0.373 & $20 / 25$ & $20 / 20$ \\
\hline & & 이 & 196 & 0.274 & 0.367 & $20 / 25$ & $20 / 20$ \\
\hline \multirow[t]{2}{*}{7} & 15 & OD & 180 & 0.375 & 0.402 & $20 / 20$ & \\
\hline & & 이 & 175 & 0.427 & 0.572 & $20 / 25$ & $20 / 20$ \\
\hline \multirow[t]{2}{*}{8} & 25 & $O D$ & 171 & 0.262 & 0.352 & $20 / 20$ & \\
\hline & & 이 & 180 & 0.223 & 0.509 & $20 / 20$ & \\
\hline \multirow[t]{2}{*}{9} & 9 & $O D$ & 180 & 0.341 & 0.511 & $20 / 25$ & $20 / 20$ \\
\hline & & 이 & 183 & 0.332 & 1.686 & $20 / 25$ & $20 / 20$ \\
\hline \multirow[t]{2}{*}{10} & 18 & OD & 185 & 0.25 & 0.334 & $20 / 20$ & \\
\hline & & 이 & 185 & 0.228 & 0.367 & $20 / 20$ & \\
\hline \multirow[t]{2}{*}{11} & 25 & OD & 222 & 0.314 & 0.411 & $20 / 25$ & $20 / 20$ \\
\hline & & 이 & 223 & 0.359 & 0.451 & $20 / 25$ & $20 / 20$ \\
\hline \multirow[t]{2}{*}{12} & 28 & OD & 230 & 0.357 & 0.595 & $20 / 20$ & \\
\hline & & 이 & 191 & 0.551 & 0.754 & $20 / 20$ & \\
\hline \multirow[t]{2}{*}{13} & 16 & $O D$ & 244 & 0.477 & 0.462 & $20 / 20$ & \\
\hline & & 이 & 241 & 0.492 & 0.53 & $20 / 20$ & \\
\hline \multirow[t]{2}{*}{14} & 16 & $O D$ & 244 & 0.498 & 0.479 & $20 / 25$ & $20 / 20$ \\
\hline & & 이 & 241 & 0.495 & 0.46 & $20 / 25$ & $20 / 20$ \\
\hline
\end{tabular}

AVSC: agudeza visual sin corrección; GMC: grosor macular central; OD: ojo derecho; OI: ojo izquierdo; ZAF: zona avascular de la fóvea.

con agujero estenopeico fue de 20/20 en los ojos con AV disminuida (Tabla 2).

\section{Discusión}

Hasta nuestro conocimiento, el presente estudio es el primero en utilizar la tecnología SS-OCT y OCT-A en pacientes con ACF en comparación con sujetos sanos en América Latina, ya que la gran mayoría de los estudios previamente publicados utilizaron la OCT de dominio espectral (SD-OCT).

Nuestro estudio reveló una disminución del GMC y un aumento de la ZAF en los pacientes con ACF comparados con el grupo de individuos sanos, mientras que el GCC y el GMP no tuvieron diferencia estadísticamente significativa entre ambos grupos. Adicionalmente se 
identificó un aplanamiento de la excavación foveal con atrofia retiniana temporal a la fóvea asociado a zonas de disminución de la densidad vascular y no perfusión focal.

En 2018, Jung, et al. ${ }^{11}$ compararon los hallazgos de SD-OCT y SS-OCT en un paciente con diagnóstico de ACF. Demostraron un adelgazamiento retiniano perifoveolar y parafoveolar temporal y nasal, con zonas de pérdida de la densidad vascular en los plexos superficial y profundo. La SS-OCT y la OCT-A permitieron una mejor visualización de las zonas perifoveales y parafoveales para diferenciar zonas de isquemia y zonas perfundidas. Se observó una mayor pérdida de la densidad vascular en la ZAF a nivel del plexo vascular profundo, lo cual se correlaciona con nuestros hallazgos en los pacientes con ACF.

Lim y $\mathrm{CaO}^{8}$ analizaron 513 ojos utilizando SD-OCT en pacientes con ACF y reportaron un promedio de GMC de $253 \mu \mathrm{m}$, frente a $262.8 \mu \mathrm{m}$ en un grupo control sano $(p=0.002)$. En nuestro estudio, la mediana del GMC fue estadísticamente inferior, pues fue de $192 \mu \mathrm{m}$ en el grupo de ACF y de $229 \mu \mathrm{m}$ en el grupo control.

En diferentes estudios publicados por Hoang, et al. ${ }^{9}$ y Han, et al. ${ }^{12}$, utilizando SD-OCT, se demuestra una disminución del GMC y se observa aplanamiento, ensanchamiento de la excavación foveal y atrofia retiniana en pacientes, que correspondían a zonas de pérdida de la densidad vascular. Atribuyeron estos hallazgos a isquemia de la red capilar foveal secundaria a oclusiones vasculares. En nuestro estudio, la OCT-A y el B-scan de la SS-OCT permitieron identificar precisamente las zonas de isquemia y pérdida de la densidad vascular en el plexo vascular profundo en 10 pacientes, que correspondían a zonas de aplanamiento foveal, atrofia retiniana temporal y disminución del GMC.

Alam, et al..$^{13}$ demostraron que el área promedio de la ZAF superficial y profunda aumentó un $53 \%$ y un $52 \%$, respectivamente, en 36 ojos de pacientes con ACF comparados con un grupo control. En nuestro estudio, la diferencia entre ambos grupos fue estadísticamente significativa, siendo el plexo profundo el de mayor compromiso, con una diferencia de mediana de $140 \mu \mathrm{m}$ entre el grupo de ACF y el grupo de personas sanas .

Mathew, et al. ${ }^{10}$ utilizaron SD-OCT y hallaron una disminución del grosor coroideo en pacientes con ACF; atribuyeron esta diferencia a la disminución de la velocidad flujo y la deformación de los drepanocitos. Sin embargo, no demostraron asociación entre los pacientes con adelgazamientos retinianos focales, lo cual podría indicar que las alteraciones coroideas son independientes de las alteraciones maculares microarteriolares. Nuestro estudio no halló diferencia estadísticamente significativa en cuanto al GCC y el GMP entre ambos grupos.

De acuerdo con los hallazgos descritos en la evaluación anatómica estructural y la AV con agujero estenopeico del grupo de ACF, podríamos teorizar que los daños estructurales alrededor de la ZAF en ambos plexos son tempranos e incipientes, sin tener afectación sobre la AV.

Es necesario confirmar la sensibilidad retiniana con pruebas de microperimetría y campo visual macular para determinar el daño funcional por dichos hallazgos.

Consideramos que las principales limitaciones del estudio fueron la pequeña muestra estudiada, que no hubo correlación de los hallazgos anatómicos con la AV mejor corregida ni con angiografía con fluoresceína, y que el grupo control sano no correspondía con edades homogéneas al grupo de ACF. Actualmente, la OCT-A presenta limitaciones inherentes a su tecnología, ya que no permite valorar la periferia extrema y está sujeta a artefactos que podrían interferir en el análisis de los resultados. Además, la medida de la ZAF se realizó manualmente, lo cual podría generar variaciones al ser dependiente del operador.

Los hallazgos descritos despiertan el interés por estudiar la relación del aumento del área de la ZAF en ambos plexos vasculares, el GMC y sus secuelas funcionales en la AV. Es necesario que los pacientes con diagnostico de ACF reciban seguimiento periódico para identificar y prevenir una progresión de la retinopatía. A medida que la tecnología avanza, la SS-OCT y la OCT-A podrían ser de gran utilidad para desarrollar protocolos de diagnóstico y seguimiento de pacientes con ACF, en especial de los jóvenes asintomáticos.

\section{Conclusión}

En comparación con el grupo control sano, el grupo con ACF tuvo menor GMC y un aumento del área de la ZAF a nivel de los plexos superficial y profundo, que fueron estadísticamente significativos, mientras que en el GCC y el GMP no lo hubo. Las imágenes de segmentación de OCT-A permitieron identificar zonas de atrofia retiniana asociadas a zonas de pérdida de densidad vascular y no perfusión. La SS-OCT y la OCT-A son novedosas modalidades de estudio no invasivas que podrían ser útiles para detectar cambios cuantitativos, microangiopáticos y estructurales en pacientes con maculopatía por ACF. Se necesitan estudios más 
extensos y a largo plazo para demostrar la relación entre los cambios de la ZAF, el GCC, el GMP y el GMC.

\section{Financiación}

El presente estudio no recibió patrocinio ni apoyo financiero de ninguna entidad para su desarrollo, autoría y publicación.

\section{Conflicto de intereses}

Los autores declaran no tener ningún conflicto de intereses.

\section{Responsabilidades éticas}

Protección de personas y animales. Los autores declaran que para esta investigación no se han realizado experimentos en seres humanos ni en animales.

Confidencialidad de los datos. Los autores declaran que han seguido los protocolos de su centro de trabajo sobre la publicación de datos de pacientes.

Derecho a la privacidad y consentimiento informado. Los autores han obtenido el consentimiento informado de los pacientes y/o sujetos referidos en el artículo. Este documento obra en poder del autor de correspondencia.

\section{Bibliografía}

1. Stuart M, Nagel R. Sickle-cell disease. Lancet. 2004;364:1343-60.

2. Elagouz M, Jyothi S, Gupta B, Sivaprasad S. Sickle cell disease and the eye: old and new concepts. Surv Ophthalmol. 2010;55:359-77.

3. Do B, Rodger D. Sickle cell disease and the eye. Curr Opin Ophthalmol. 2017;28:623-8.

4. Misnaza Castrillón SP. Drepanocitosis en Colombia: análisis de la notificación como enfermedad huérfana o rara al sistema de vigilancia en salud pública, 2016 y 2017; 23:1-13. Disponible en: https://www. ins.gov.co/.

5. Hassell K. Population estimates of sickle cell disease in the U.S. Am J Prevent Med. 2010;38:S512-21.

6. Stevens T. Sickling hemoglobinopathies. Arch Ophthalmol. 1974;92:455.

7. Ryan S. Occlusion of the macular capillaries in sickle cell hemoglobin $\mathrm{C}$ disease. Am J Ophthalmol. 1974;77:459-61.

8. Lim J, Cao D. Analysis of retinal thinning using spectral-domain optical coherence tomography imaging of sickle cell retinopathy eyes compared to age- and race-matched control eyes. Am J Ophthalmol. 2018;192:229-38.

9. Hoang Q, Chau F, Shahidi M, Lim J. Central macular splaying and outer retinal thinning in asymptomatic sickle cell patients by spectral-domain optical coherence tomography. Am J Ophthalmol. 2011:151:990-4.e1.

10. Mathew R, Bafiq R, Ramu J, Pearce E, Richardson M, Drasar E, et al. Spectral domain optical coherence tomography in patients with sickle cell disease. Br J Ophthalmol. 2015;99:967-72.

11. Jung J, Chen M, Frambach C, Rofagha S, Lee S. Spectral domain versus swept source optical coherence tomography angiography of the retinal capillary plexuses in sickle cell maculopathy. Retin Cases Brief Rep. 2018:12:87-92.

12. Han I, Tadarati M, Scott A. Macular vascular abnormalities identified by optical coherence tomographic angiography in patients with sickle cell disease. JAMA Ophthalmol. 2015;133:1337.

13. Alam M, Thapa D, Lim J, Cao D, Yao X. Quantitative characteristics of sickle cell retinopathy in optical coherence tomography angiography. Biomed Opt Express. 2017;8:1741-53 